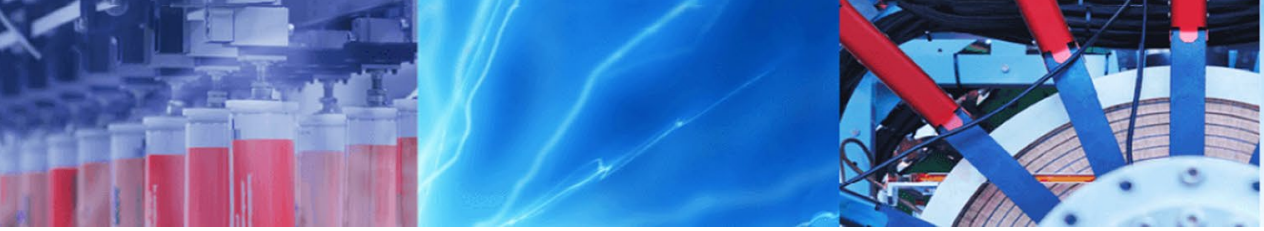

Research Article

\title{
Metamaterials with Poisson's ratio sign toggling by means of microstructural duality
}

\author{
Teik-Cheng $\operatorname{Lim}^{1}$ (1)
}

(c) Springer Nature Switzerland AG 2019

\begin{abstract}
Neither positive nor negative Poisson's ratio materials are fully advantageous across a wide range of load bearing applications. In some cases, it is advantageous for structures to possess duality in material properties so as to take advantage of auxetic behavior under one loading condition as well as conventional behavior when the loading condition changes. This paper proposes two metamaterials - the hybrid rhombic-re-entrant metamaterial and the hybrid kite-arrowhead metamaterial-in which the prescription of either compressive or tensile load always leads to lateral expansion. Specifically, the Poisson's ratio of these metamaterials range from negative infinity to zero upon tensile load, and from zero to positive infinity upon compressive load. This duality of auxetic and conventional behavior for each of these metamaterial is achieved by employing a simultaneous lock and slide mechanism that is interchangeable upon load reversal. When loaded in one direction, the locked part takes effect while the sliding part becomes redundant. Upon load direction reversal, the previously locked and sliding parts convert to sliding and locked parts, respectively, thereby activating the redundant part while deactivating the functional part. The change in effective microstructural components, therefore, facilitates Poisson's ratio sign toggling with load reversal. Due to the indiscriminate lateral expansion regardless of the axial loading direction, such behavior in a fiber is useful to resist both fiber pull-out as well as fiber push-out from the matrix material.
\end{abstract}

Keywords Auxetic $\cdot$ Conventional $\cdot$ Metamaterials $\cdot$ Microstructural duality

\section{Introduction}

Under the influence of an applied tensile or compressive normal stress, and hence a prescribed normal strain $\varepsilon_{n}$, common materials tend to contract or expand, respectively, in the direction orthogonal to the load application, e.g. the transverse strain $\varepsilon_{t}$ possesses a sign that is opposite to that of $\varepsilon_{n}$. See Fig. 1a. Suppose a material property is defined as being $\varepsilon_{t} / \varepsilon_{n}$, then most list of such material properties would give a predominantly, or even completely, negative values. For this reason, the Poisson's ratio has been defined in such a manner so as to provide positive values, i.e. $v=-\varepsilon_{t} / \varepsilon_{n}$. The belief on the positivity of the Poisson's ratio for isotropic solids, at the expense on its negativity, has been so prevalent that almost all publications-be they data handbooks, archival journal papers, textbooks, technical reports, etc.-furnish mechanical and other physical responses for positive Poisson's ratio within the range $0 \leq v \leq 0.5$, and mainly $v=0.3$ if a typical value is needed or about $v=0.2$ in some cases. Interest was therefore generated whenever materials of negative Poisson's ratio-illustrated in Fig. $1 \mathrm{~b}$-were reported (e.g. [1-7]), although such negativity should not be a total surprise since it has been proven earlier that the Poisson's ratio falls within the range $-1 \leq v \leq 0.5$ for isotropic solids $[8,9]$; in the case of anisotropic solids, it was subsequently proven that their Poisson's ratio has no bounds $[10,11]$. The term "auxetic" materials was coined in 1991 [12], thus

Teik-Cheng Lim, alan_tc_lim@yahoo.com | 'School of Science and Technology, Singapore University of Social Sciences, 463 Clementi Road, Singapore 599494, Singapore.

SN Applied Sciences (2019) 1:176| https://doi.org/10.1007/s42452-019-0185-1

Received: 21 November 2018 / Accepted: 16 January 2019 / Published online: 22 January 2019 


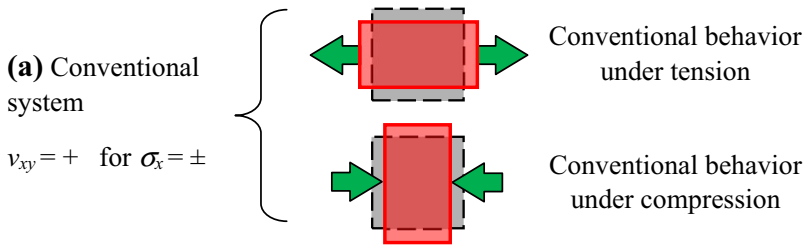

(b) Auxetic system
$v_{x y}=-$ for $\sigma_{x}= \pm$
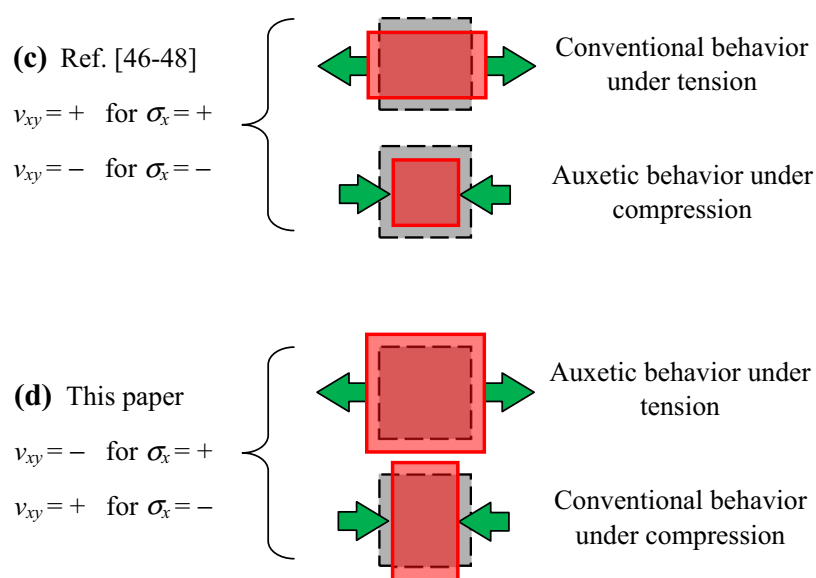

Fig. 1 Visual description of various materials under uniaxial load: a conventional materials, b auxetic materials, $\mathbf{c}$ materials that exhibit conventional behavior under tension but auxetic under compression [46-48], and d materials that exhibit auxetic behavior under tension but conventional behavior under compression (this paper)

auxeticity refers to the extent of negativity of Poisson's ratio. Applications of auxetic materials and structures have been widely explored due to their rare characteristic, without which it would be technically impossible to obtain [13]. These include, but not limited to, piezocomposites and piezoelectric sensors [14, 15], filters [16, 17], artificial blood vessels [18], fasteners [19], deployable antenna for deep space missions [20] and technical textiles [21]. Research in auxetics has been expanding exponentially such that a comprehensive literature survey would be too voluminous, hence interested readers are referred to reviews [22-44] and a monograph [45] in this field.

It is obvious that the Poisson's ratio signs for both conventional and auxetic materials are retained regardless of the direction of loading direction. However, greater flexibility in design of materials and structures can be achieved if material properties can be reversed under the influence of reversed loading. For example, one may wish to design a beam that always contracts laterally whenever an axial stress is applied along the beam, regardless of whether the axial stress is tensile or compressive. To achieve this, the beam material must possess a positive Poisson's ratio under tensile load, but becomes an auxetic material under compressive load. One such microstructure is that of centro-symmetric honeycombs with T-shaped joints [46], which consists of a single material, but with differing rib thicknesses, as shown in Fig. 2a. An alternative to this can be achieved by means of composites with pin-jointed rods of equal, or almost equal, thicknesses but of different material moduli-long flexible rods and short rigid rods indicated by red and blue, respectively, in Fig. $2 b$ - which is known as rectangular-cell in triangular-array microstructure $[47,48]$. Reference to Fig. $2 a$, b indicate that the application tensile and compressive loads in the direction of the short rods leads to transformation of the rectangular cells into hexagonal and re-entrant cells, respectively. A similar effect can be observed for the triangular-cell in rectangular-array microstructure illustrated in Fig. 2c whereby application of tensile and compressive loads in the same direction converts the triangular cell into kite and double arrowhead cells, respectively $[47,48]$.

What if there is a need to produce a beam that expands laterally under the influence of axial load, regardless of whether the axial load is tensile or compressive? For such a material, the Poisson's ratio must be negative under axial stretching but the Poisson's ratio must become positive when the axial load is compressive. An idealized schematic for contrasting the sign switching with load reversal considered herein with a recent approach [46-48] is furnished in Fig. 3 based on $\sigma_{x}$ loading; in addition, diagrams for conventional and auxetic materials are included for the sake of completeness. The choice of negativity in the vertical axis is made so as to give positive and negative slopes corresponding to positive and negative $v_{x y}$, respectively. The property shown in Fig. $3 \mathrm{~d}$ is attained herein by using the hybrid rhombic-re-entrant metamaterial and the hybrid kite-arrowhead metamaterial discussed in Sects. 2.2 and 2.3 , respectively.

\section{Analysis}

\subsection{Preamble}

The basic hybrid rhombic-re-entrant metamaterial microstructure is shown Fig. 4a, which consists of a pair of slot and slider to effect simultaneous jam and slide mechanism. Each column consists of a zigzag structure with alternating slots and sliders pointing to opposite directions, as shown in Fig. 4b, wherein the slots are joined to the sliders in a neighboring zigzag structure while the sliders are joined 
Fig. 2 Microstructures that exhibit lateral contraction with axial tension or compression: a centro-symmetric honeycombs with T-shaped joints [46], b rectangular-cell in triangular-array microstructure $[47,48]$, and c triangular-cell in rectangulararray microstructure $[47,48]$. The microstructure (a) is made from a single material with differing thicknesses, while those of $\mathbf{b}$ and $\mathbf{c}$ are composites with pin-jointed rods of contrasting moduli

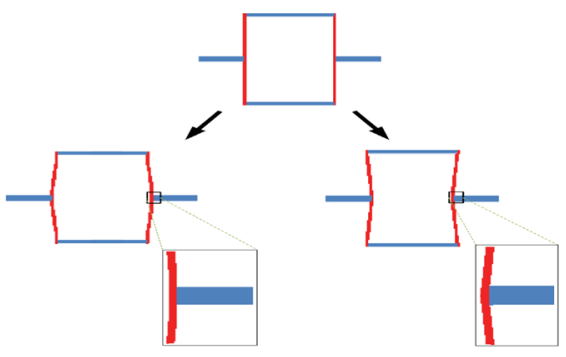

(a)
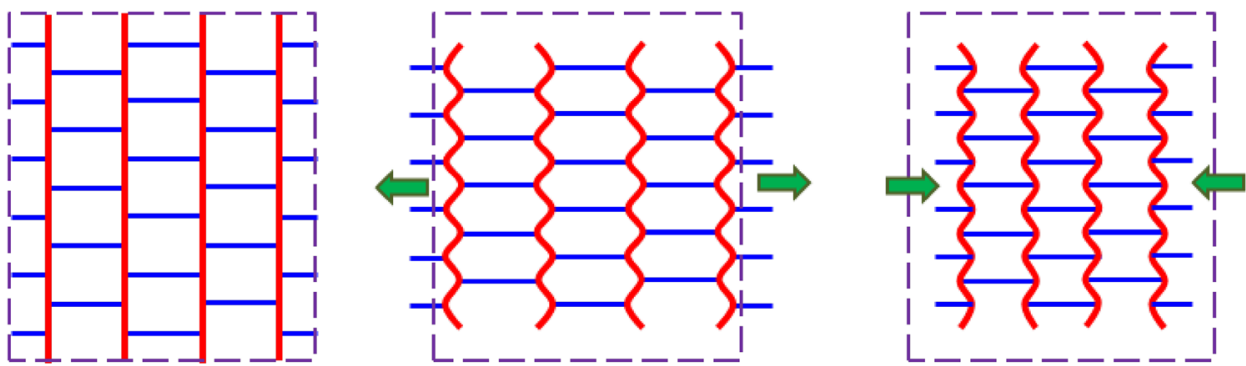

(b)
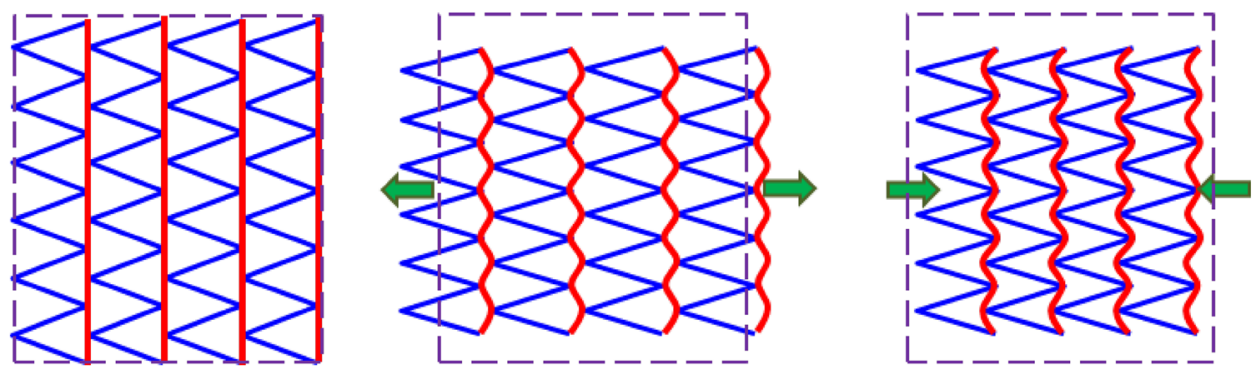

(c) to the slots in another neighboring zigzag structure. The zigzag vertices are pin-joints to permit free rotations with each rod member being rigid. In the relaxed state, i.e. original state as indicated in Fig. 4b, the sliders lightly touch the open end of the slots while the vertices from neighboring zigzags also lightly touch one another. Upon application of compressive stress in the x-direction, as shown in Fig. 4c, the vertices are prevented from motion along the $x$-direction due to symmetric constraints from neighboring vertices and therefore negative strain in $\mathrm{x}$-direction is made possible through motion of the sliders along the slots towards the closed ends, and a simultaneous rotation of the inclined rods. In other words, the contact points of the vertices are effectively pin joints while the slot-slider pairs are effectively non-structural. The effective microstructure is hence that of rhombic geometry, which is a special case of hexagonal honeycomb with diminished horizontal ribs. When a tensile stress is applied in the $\mathrm{x}$-direction as indicated in Fig. $4 d$, the interlock between the sliders and the open ends of the slots retains the total dimension of the horizontal parts such that positive strain in $\mathrm{x}$-direction is made possible through rotation of the inclined rods. The slot-slider pairs effectively become rigid horizontal rods and the effective microstructure is therefore that of reentrant geometry. In other words, the hybrid rhombic-reentrant metamaterial exhibits microstructural duality, and hence behavioural duality with opposing Poisson's ratio signs for opposing stress directions.

A unit of the hybrid kite-arrowhead metamaterial microstructure is illustrated in Fig. 5a, each consisting of two slots and two sliders. The inner and outer sliders are placed in the inner and outer slots, respectively, of the neighboring parts, as illustrated in Fig. $5 \mathrm{~b}$. In this original state, both the sliders are in light contact with the closed end of the slots. The outer slot-slider pairs are locked with the application of compressive stress in the $x$-direction, while the inner sliders move toward the open ends of their corresponding slots, as denoted in Fig. 5c. Essentially, the outer slot-slider pairs form rigid rods while the inner slot-slider pairs are non-structural so as to effectively form a network of kite microstructural geometry. When tensile load is applied in the $x$-direction as shown in Fig. $5 d$, the inner slot-slider pairs are being locked, while the outer sliders move toward the open 


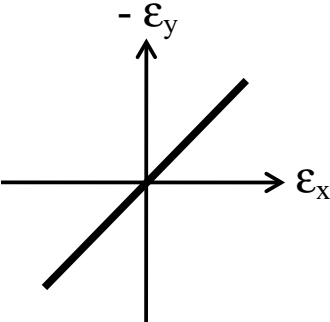

(a)

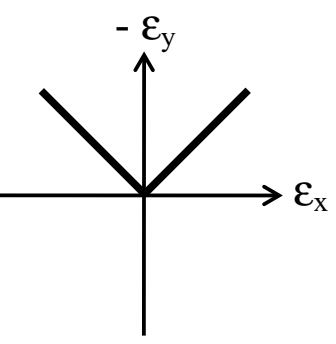

(c)

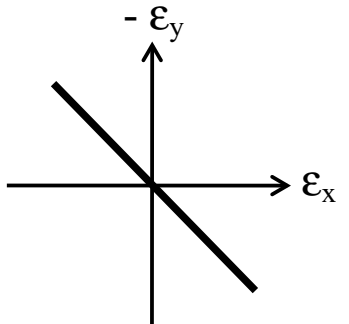

(b)

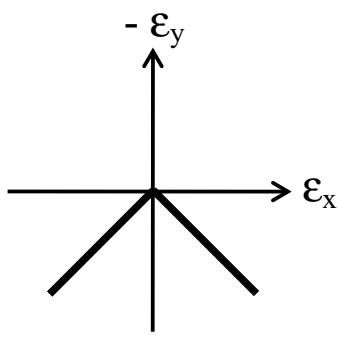

(d)
Fig. 3 Idealized schematics using the $-\varepsilon_{y}$ versus $\varepsilon_{x}$ plane under $\sigma_{x}$ loading for describing a conventional materials, $\mathbf{b}$ auxetic materials, c materials with Poisson's ratio sign toggling with $\sigma_{x}$ reversal covered in Refs. [46-48], and $\mathbf{d}$ metamaterials with opposite sense of Poisson's ratio sign toggling in this paper

ends of the outer slots. The inner slot-slider pairs become rigid rods while the outer slot-slider pairs are non-load bearing. This results in a modified double arrowhead geometry. For this reason, it can be said that the hybrid kite-arrowhead metamaterial demonstrates microstructural duality, and therefore behavioural duality with opposite Poisson's ratio signs being manifested under opposing stress directions.

In what follows, the representative units for both metamaterials' microstructures are isolated for the purpose of analysis, whereby the origin is denoted by " $\mathrm{O}$ " while the vertices " $A$ " and " $B$ " are freely rotating pin-jointed vertices as indicated in Figs. 6 and 7. For the hybrid rhombic-reentrant microstructure, "O" is located at the mid-span of a horizontal slot-slider mechanism whereas " $\mathrm{C}$ " is located midway in the nearest horizontal slot-slider; both are not freely rotating pin-joints for this microstructure. For the hybrid kite-arrowhead microstructure, " $\mathrm{O}$ " is a freely-rotating joint while " $\mathrm{C}$ " is also a freely rotating joint, but forms the origin of the neighbouring representative unit. The original state of both microstructures are shown in Figs. $6 a$ and $7 \mathrm{a}$. The geometries illustrated in Figs. $6 \mathrm{~b}$ and $7 \mathrm{~b}$ refer to the case where compressive stress is applied along the $\mathrm{x}$-direction, while those in Figs. $6 \mathrm{c}$ and $7 \mathrm{c}$ correspond to the case where tensile stress is applied in the $\mathrm{x}$-direction.

\subsection{Hybrid rhombic-re-entrant metamaterial}

The analysis of compression in $\mathrm{x}$-direction for the hybrid rhombic-re-entrant metamaterial can be established by comparing Fig. $6 \mathrm{~b}$ against Fig. $6 \mathrm{a}$, in which $\mathrm{B}$ is prevented from moving to the left due to a symmetrically opposing vertex; overall contraction in $x$-direction is therefore made possible with the sliding of vertices $A$ and $C$ in the slots towards $O$ and $B$, respectively, to their new locations $A^{\prime}$ and $C^{\prime}$, with the vertex $B$ shifting upward to $B^{\prime}$. This causes the inclined rod of length / to rotate clockwise by an amount $d \theta$ such that the projections $y_{0}$ elongates to $y$ while $x_{0}$ shortens to $x$.

From the projected dimension along the $y$-axis

$y_{0}=/ \sin \theta$

in the original state, and

$y=I \sin (\theta+d \theta) \approx I(\sin \theta+d \theta \cos \theta)$

in the deformed state, we have the change in projected dimension along y-direction

$d y=y-y_{0}=I d \theta \cos \theta$

so as to give the corresponding strain component

$\varepsilon_{y}=\frac{d y}{y_{0}}=\frac{d \theta}{\tan \theta}$.

Based on the projected dimension along the $\mathrm{x}$-axis

$x_{0}=l \cos \theta$

in the original state, and

$x=I \cos (\theta+d \theta) \approx I(\cos \theta-d \theta \sin \theta)$

in the deformed state, one obtains the change of projected dimension in $\mathrm{x}$-direction

$d x=x-x_{0}=-l d \theta \sin \theta$

to yield its strain component

$\varepsilon_{x}=\frac{d x}{x_{0}}=-d \theta \tan \theta$

This gives the Poisson's ratio for the hybrid rhombic-reentrant microstructure for $\sigma_{x}<0$

$v_{x y}=-\frac{\varepsilon_{y}}{\varepsilon_{x}}=\frac{1}{\tan ^{2} \theta}$,

which indicates conventional behavior for $\sigma_{x}<0$.

The analysis of tension in $\mathrm{x}$-direction for the hybrid rhombic-re-entrant metamaterial can be obtained by comparing Fig. 6c vis-à-vis Fig. 6a. Since the sliders are locked on the right side of the slots, extension in x-direction is made possible by clockwise rotation of the inclined rod by an angle $d \theta$ such that $B C$ undergoes curvilinear motion to $B^{\prime} C^{\prime}$, i.e. $B^{\prime} C^{\prime}$ remains horizontal and retains the 
Fig. 4 The hybrid rhombic-reentrant metamaterial microstructure, showing a a slot and a slider in a symmetrical half, b original microstructure, $\mathbf{c}$ conversion to rhombic microstructure under compressive load, and $\mathbf{d}$ conversion to reentrant microstructure under tensile load. The effective parts in the middle units of $\mathbf{c}$ and $\mathbf{d}$ are indicated in grey. A dashed rectangle that encompasses the microstructure (b) is transposed on $\mathbf{c}$ and $\mathbf{d}$ to aid visual comparison

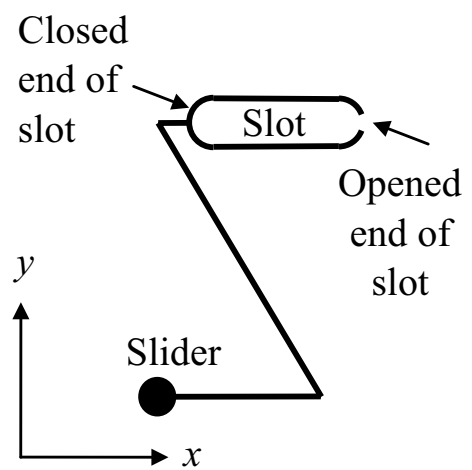

(a)

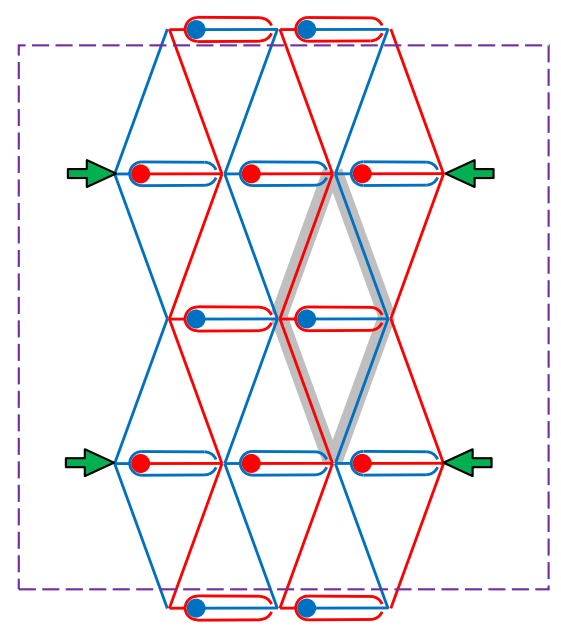

(c)

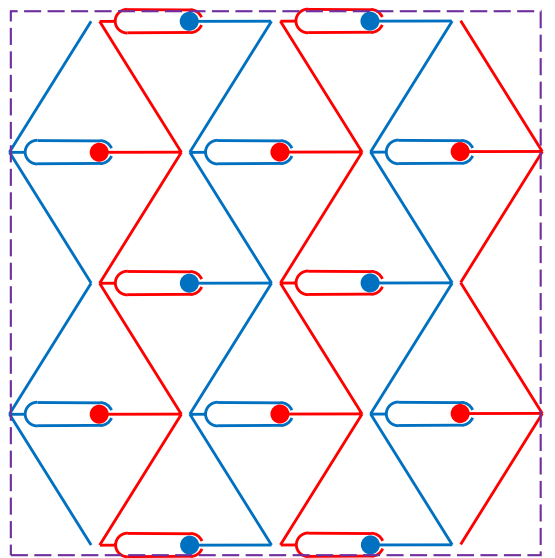

(b)

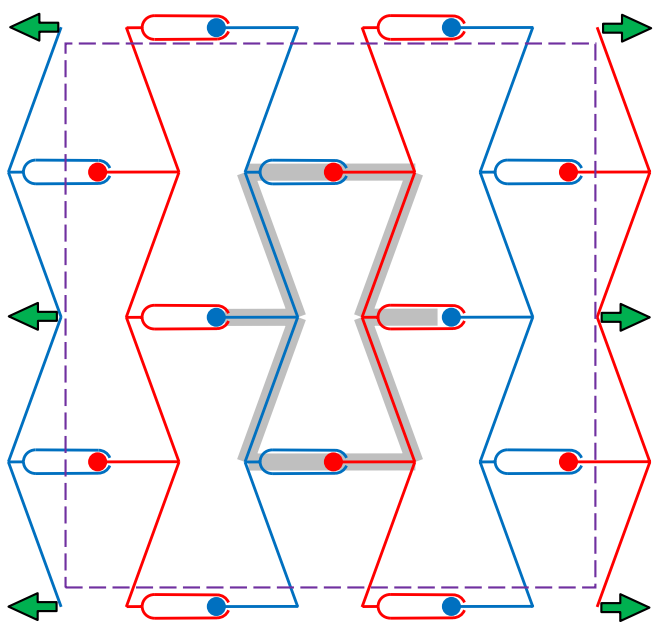

(d) same length as that for BC. Considering the original state, the projected length on $y$-axis is given by Eq. (1) and, for a clockwise rotation of $d \theta$ the projected length at the same axis is the same as that in Eq. (2), thereby leading to the incremental change of dimension in y-direction and its strain as furnished in Eqs. (3) and (4), respectively. Likewise, the projected dimension along the $\mathrm{x}$-axis in the original state is that given in Eq. (5). Upon rotation of the inclined rod by $d \theta$, the projected dimension along the x-direction can be obtained from Fig. $6 \mathrm{c}$ as

$x=2 x_{0}-I \cos (\theta+d \theta) \approx I(\cos \theta+d \theta \sin \theta)$

so as to give

$d x=x-x_{0}=l d \theta \sin \theta$

and its corresponding strain

$\varepsilon_{x}=\frac{d x}{x_{0}}=d \theta \tan \theta$ $v_{x y}=-\frac{\varepsilon_{y}}{\varepsilon_{x}}=-\frac{1}{\tan ^{2} \theta}$

which indicates auxetic behavior for $\sigma_{x}>0$.

\subsection{Hybrid kite-arrowhead metamaterial}

The analysis of compression in $\mathrm{x}$-direction for the hybrid kite-arrowhead metamaterial can be established by contrasting Fig. $7 b$ with reference to Fig. 7a, whereby the length $I_{3}$ remains constant as $A$ and $C$ displace to $A^{\prime}$ and $C^{\prime}$, respectively. During this time, $O A$ rotates anticlockwise to $O A^{\prime}$ by an angle $d \theta_{1}$ while $A C$ rotates clockwise to $A^{\prime} C^{\prime}$ by $d \theta_{3}$. Therefore, the compression analysis considers the movement of linkage $O A C$ while $A B C$ is redundant.

Perusal to Fig. 7a for the projection on $y$-axis

$y_{0}=I_{1} \sin \theta_{1}=I_{3} \sin \theta_{3}$

thereby leading to 
Fig. 5 The hybrid kite-arrowhead metamaterial microstructure, showing a a pair of slots and a pair of sliders in a symmetrical half, $\mathbf{b}$ original microstructure, c conversion to kite microstructure under compressive load, and $\mathbf{d}$ conversion to double arrowhead-like microstructure under tensile load. The effective parts in the middle units of $\mathbf{c}$ and $\mathbf{d}$ are indicated in grey. A dashed rectangle that encompasses the original microstructure (b) is transposed on $\mathbf{c}$ and $\mathbf{d}$ to aid visual comparison

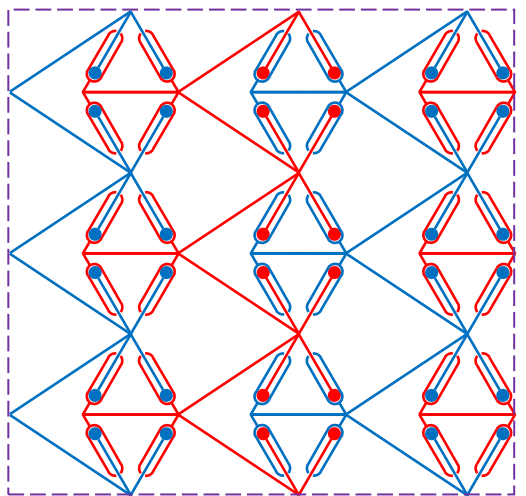

(b)

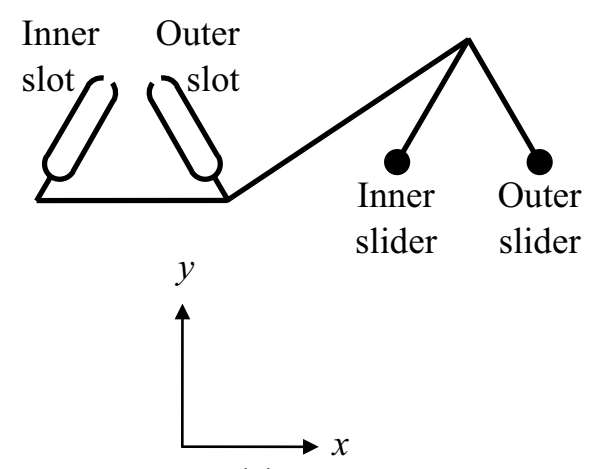

(a)

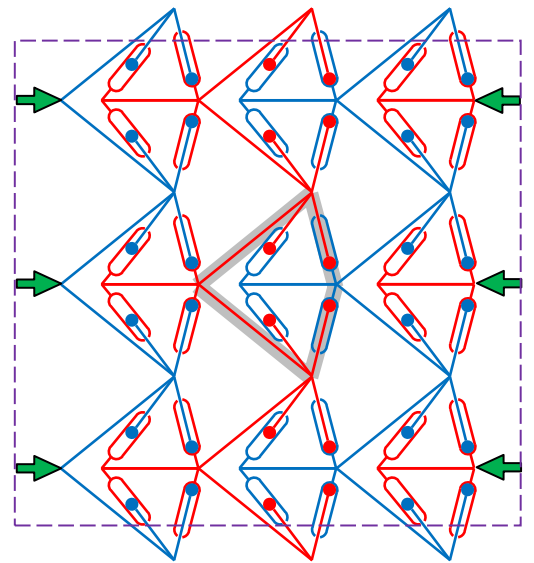

(c)

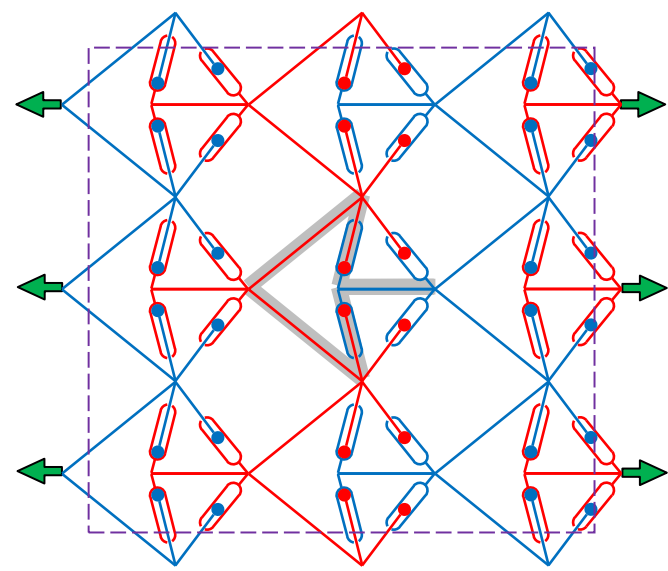

(d) at the original state, and consideration of $y=I_{1} \sin \left(\theta_{1}+d \theta_{1}\right)=I_{3} \sin \left(\theta_{3}+d \theta_{3}\right)$ from Fig. $7 \mathrm{~b}$ for similar projection gives

$y=I_{1}\left(\sin \theta_{1}+d \theta_{1} \cos \theta_{1}\right)=I_{3}\left(\sin \theta_{3}+d \theta_{3} \cos \theta_{3}\right)$

based on infinitesimal deformation. This gives the incremental change in dimension measured along the $y$-axis

$d y=y-y_{0}=I_{1} d \theta_{1} \cos \theta_{1}=I_{3} d \theta_{3} \cos \theta_{3}$

and its corresponding strain

$\varepsilon_{y}=\frac{d y}{y_{0}}=\frac{d \theta_{1}}{\tan \theta_{1}}=\frac{d \theta_{3}}{\tan \theta_{3}}$.

To pave a way for obtaining the strain in $\mathrm{x}$-direction, it is useful at this stage to write

$\frac{l_{3}}{l_{1}}=\frac{\sin \theta_{1}}{\sin \theta_{3}}$

based on Eq. (14), and

$\sin \theta_{1}+d \theta_{1} \cos \theta_{1}=\frac{l_{3}}{l_{1}}\left(\sin \theta_{3}+d \theta_{3} \cos \theta_{3}\right)$

from Eq. (15). Substituting Eq. (18) into Eq. (19) gives $\frac{d \theta_{3}}{d \theta_{1}}=\frac{\tan \theta_{3}}{\tan \theta_{1}}$.

Reference to Fig. 7a gives the projection along the $x$-axis through OAC as $x_{0}=I_{1} \cos \theta_{1}+I_{3} \cos \theta_{3}$ or, using Eq. (18),

$x_{0}=I_{1}\left(\cos \theta_{1}+\frac{\sin \theta_{1}}{\tan \theta_{3}}\right)$

for the original state, while perusal to Fig. $7 b$ yields a similar projection through $O A^{\prime} C^{\prime}$ as $x=l_{1} \cos \left(\theta_{1}+d \theta_{1}\right)+l_{3} \cos \left(\theta_{3}+d \theta_{3}\right)$, or

$x=I_{1}\left(\cos \theta_{1}-d \theta_{1} \sin \theta_{1}\right)+I_{3}\left(\cos \theta_{3}-d \theta_{3} \sin \theta_{3}\right)$

on the basis of infinitesimal rod rotation. Substituting Eqs. (18) and (20) into Eq. (22) eliminates $I_{3}$ and $d \theta_{3}$ to yield $x=I_{1}\left(\cos \theta_{1}-d \theta_{1} \sin \theta_{1}+\frac{\sin \theta_{1}}{\tan \theta_{3}}-\cos \theta_{1} \tan \theta_{3} d \theta_{1}\right)$.

Therefore, we have the incremental change in dimension along the $x$-direction

$d x=x-x_{0}=-l_{1} d \theta_{1}\left(\sin \theta_{1}+\cos \theta_{1} \tan \theta_{3}\right)$

\section{SN Applied Sciences}




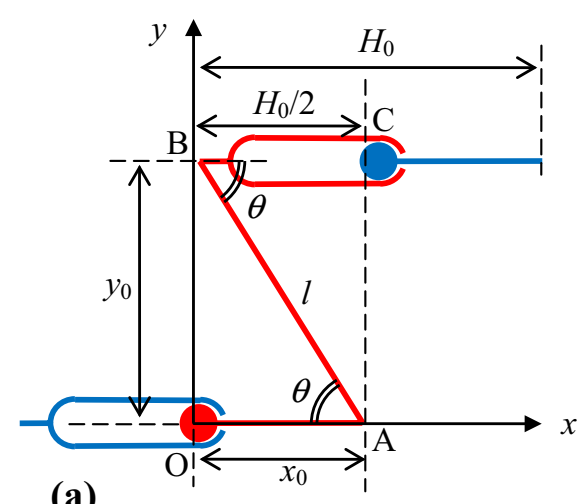

(a)

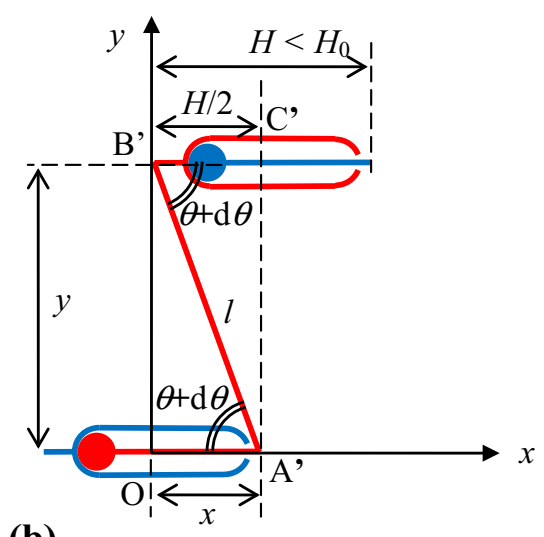

(b)

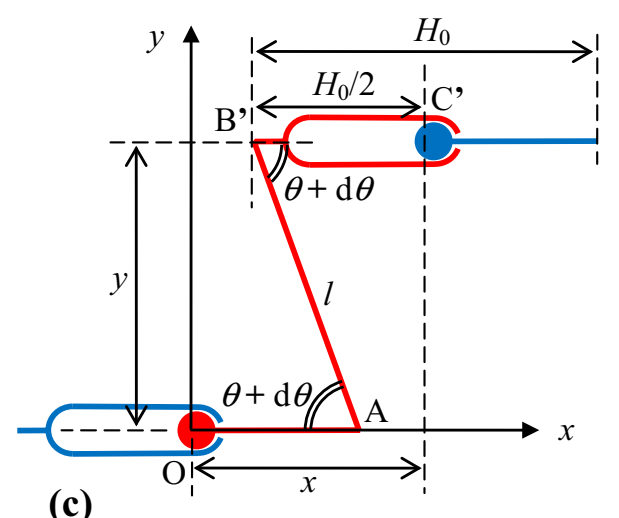

Fig. 6 A representative unit of the hybrid rhombic-re-entrant metamaterial for analysis: a original state, b compressed, and c stretched along $\mathrm{x}$-direction

and strain in the same direction

$\varepsilon_{x}=\frac{d x}{x_{0}}=-d \theta_{1} \tan \theta_{3}$

This gives the Poisson's ratio

$v_{x y}=-\frac{\varepsilon_{y}}{\varepsilon_{x}}=\frac{1}{\tan \theta_{1} \tan \theta_{3}}$

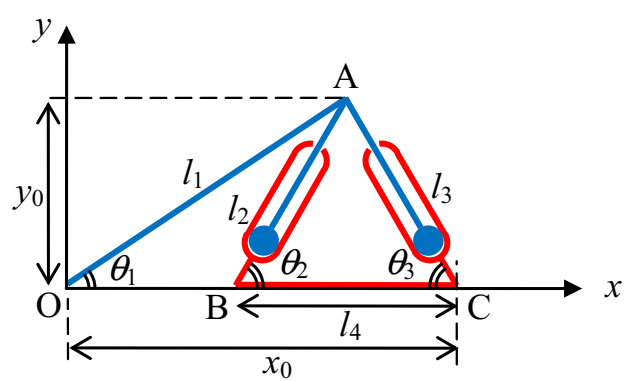

(a)

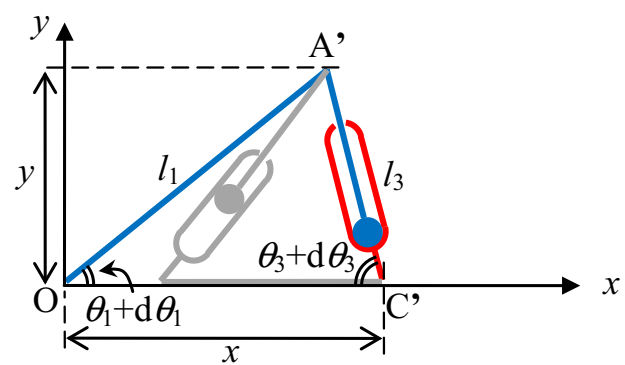

(b)

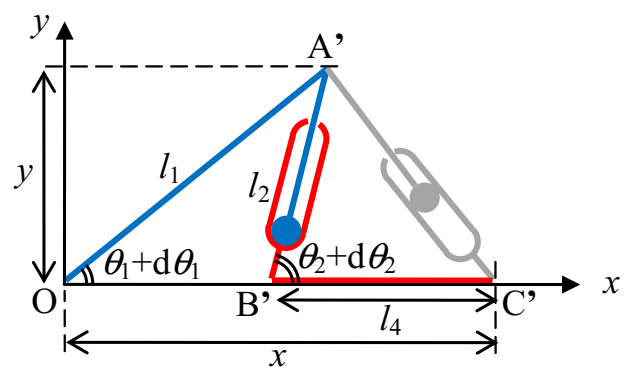

(c)

Fig. 7 A representative unit of the hybrid kite-arrowhead metamaterial for analysis: a original state, b compressed, and c stretched along $\mathrm{x}$-direction. Redundant parts are indicated in grey

which denotes conventional behavior for $\sigma_{x}<0$. For the special case where $I_{1}=I_{3}\left(\operatorname{or} \theta_{1}=\theta_{3}\right)$, then Eq. (26) simplifies to

$v_{x y}=\frac{1}{\tan ^{2} \theta_{1}}$,

which is analogous to Eq. (9) for the compression of the hybrid rhombic-re-entrant microstructure in the $x$-direction.

The analysis of tensile load application in $\mathrm{x}$-direction on the hybrid kite-arrowhead metamaterial is carried out by comparing Fig. $7 c$ with respect to Fig. $7 a$, whereby the length $I_{2}$ remains constant as $A, B$ and $C$ displace to $A^{\prime}, B^{\prime}$ and $C^{\prime}$, respectively. During this time, $O A$ and $A B$ rotate anticlockwise to $O A^{\prime}$ and $A^{\prime} B^{\prime}$ by $d \theta_{1}$ and $d \theta_{2}$, respectively, while $B C$ moves to $B^{\prime} C^{\prime}$ by translation along the $x$-axis. In 
other words, the tensile analysis accounts for the motion of linkage $O A B C$, with $A C$ being redundant. Perusal to Fig. 7a for the projection on $y$-axis at the original state

$y_{0}=I_{1} \sin \theta_{1}=I_{2} \sin \theta_{2}$

and consideration of $y=I_{1} \sin \left(\theta_{1}+d \theta_{1}\right)=I_{2} \sin \left(\theta_{2}+d \theta_{2}\right)$ from Fig. $7 \mathrm{c}$ for similar projection gives rise to

$y=I_{1}\left(\sin \theta_{1}+d \theta_{1} \cos \theta_{1}\right)=I_{2}\left(\sin \theta_{2}+d \theta_{2} \cos \theta_{2}\right)$

on the assumption of infinitesimal deformation. This gives the incremental change in dimension measured along the $y$-axis

$d y=y-y_{0}=I_{1} d \theta_{1} \cos \theta_{1}=I_{2} d \theta_{2} \cos \theta_{2}$

and its strain

$\varepsilon_{y}=\frac{d y}{y_{0}}=\frac{d \theta_{1}}{\tan \theta_{1}}=\frac{d \theta_{2}}{\tan \theta_{2}}$.

As before, it is beneficial to express

$\frac{l_{2}}{l_{1}}=\frac{\sin \theta_{1}}{\sin \theta_{2}}$

based on Eq. (28) and

$\sin \theta_{1}+d \theta_{1} \cos \theta_{1}=\frac{l_{2}}{l_{1}}\left(\sin \theta_{2}+d \theta_{2} \cos \theta_{2}\right)$

from Eq. (29). Substituting Eq. (32) into Eq. (33) gives

$\frac{d \theta_{2}}{d \theta_{1}}=\frac{\tan \theta_{2}}{\tan \theta_{1}}$.

In addition,

$I_{4}=I_{2} \cos \theta_{2}+I_{3} \cos \theta_{3}$.

Reference to Fig. 7 a gives the projection along the $x$-axis through $O A B C$ as $x_{0}=I_{1} \cos \theta_{1}-I_{2} \cos \theta_{2}+I_{4}$ or, using Eq. (32),

$x_{0}=I_{1}\left(\cos \theta_{1}-\frac{\sin \theta_{1}}{\tan \theta_{2}}\right)+I_{4}$

for the original state, while perusal to Fig. 7c yields a similar projection through $O A^{\prime} B^{\prime} C^{\prime}$ as $x=I_{1} \cos \left(\theta_{1}+d \theta_{1}\right)-I_{2} \cos \left(\theta_{2}+d \theta_{2}\right)+I_{4}$, or

$x=I_{1}\left(\cos \theta_{1}-d \theta_{1} \sin \theta_{1}\right)-I_{2}\left(\cos \theta_{2}-d \theta_{2} \sin \theta_{2}\right)+I_{4}$

based on infinitesimal rod rotation. Substituting Eqs. (32) and (34) into Eq. (37) eliminates $I_{2}$ and $d \theta_{2}$ to yield

$x=I_{1}\left(\cos \theta_{1}-d \theta_{1} \sin \theta_{1}-\frac{\sin \theta_{1}}{\tan \theta_{2}}+\cos \theta_{1} \tan \theta_{2} d \theta_{1}\right)+I_{4}$.

Therefore, we have the incremental change in dimension along the $x$-direction $d x=x-x_{0}=I_{1} d \theta_{1}\left(-\sin \theta_{1}+\cos \theta_{1} \tan \theta_{2}\right)$

and strain in the corresponding direction

$\varepsilon_{x}=\frac{d x}{x_{0}}=\frac{d \theta_{1} \tan \theta_{2}\left(\frac{1}{\tan \theta_{1}}-\frac{1}{\tan \theta_{2}}\right)}{\frac{1}{\tan \theta_{1}}-\frac{1}{\tan \theta_{2}}+\frac{I_{4}}{l_{1} \sin \theta_{1}}}$.

Substituting Eq. (35) into Eq. (40) gives

$\varepsilon_{x}=d \theta_{1} \tan \theta_{3} \frac{\tan \theta_{2}-\tan \theta_{1}}{\tan \theta_{3}+\tan \theta_{1}}$

thereby leading to the Poisson's ratio

$v_{x y}=-\frac{\varepsilon_{y}}{\varepsilon_{x}}=\frac{1}{\tan \theta_{1} \tan \theta_{3}}\left(\frac{\tan \theta_{1}+\tan \theta_{3}}{\tan \theta_{1}-\tan \theta_{2}}\right)$,

which indicates auxetic behavior for $\sigma_{x}>0$ because $\tan \theta_{1}<\tan \theta_{2}$. Although AC is redundant in the case of tensile $\sigma_{x}$, the angle $\theta_{3}$ comes into play arising from the need to include the length $I_{4}$ for evaluating $x$, i.e. $O C^{\prime}$, in Fig. 7c. Equation (42) is comparable to Eq. (26) except for the terms in the parenthesis.

\section{Results and discussion}

\subsection{Experimental}

A unit cell for each metamaterial has been constructed in such a manner that permits combined rotation and sliding motion, as shown in Figs. 8 and 9 for the hybrid rhombic-re-entrant and the hybrid kite-arrowhead metamaterials, respectively. For both unit cells, the top picture refers to the unit cell in its original condition before deformation. The remaining pictures on the left, i.e. (b), (d) and (f), denote compression while those on the right, i.e. (c), (e) and (g), indicate tension along the horizontal direction. The experimental strains were calculated based on the center of the hinges for indicating the unit cell boundaries. The theoretical $\varepsilon_{y}$ of the hybrid rhombic-reentrant unit cell were calculated using Eqs. (9) and (13) for the prescribed decrease and increase, respectively, of the cell dimension in x-direction, while the theoretical $\varepsilon_{y}$ of the hybrid kite-arrowhead unit cell were computed from Eqs. (26) and (42) for the imposed decrease and increase, respectively, of the cell dimension in $x$-direction. Comparison of the theoretical and experimental strains in Fig. 10 reveal that better agreement is shown for the hybrid rhombic-re-entrant unit cell due to the lesser amount of linkages and joints. The greater number of linkages and joints for the hybrid kite-arrowhead unit cell leads to more unintended slippage at the rotating joints, and hence more 


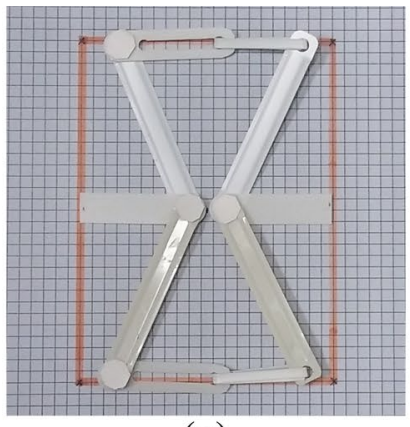

(a)

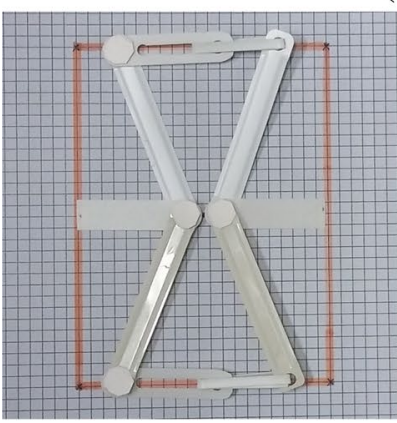

(b)

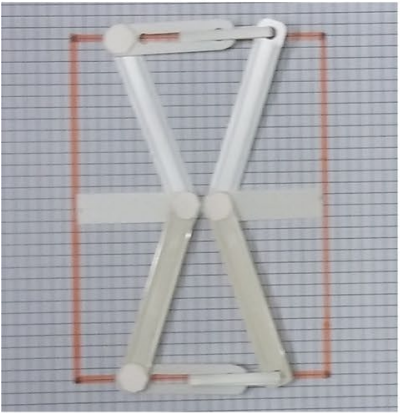

(d)

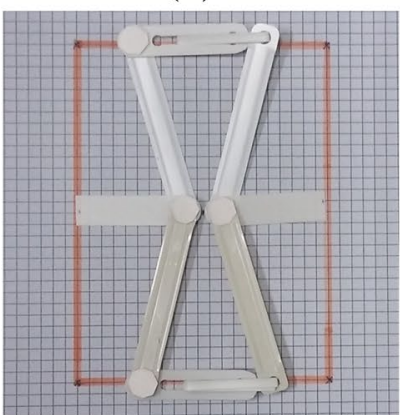

(f)

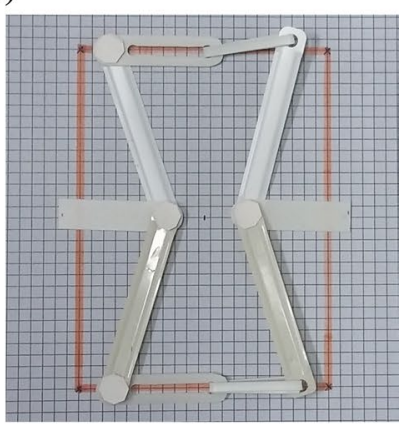

(c)

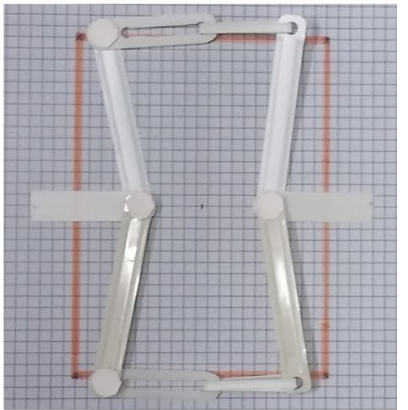

(e)

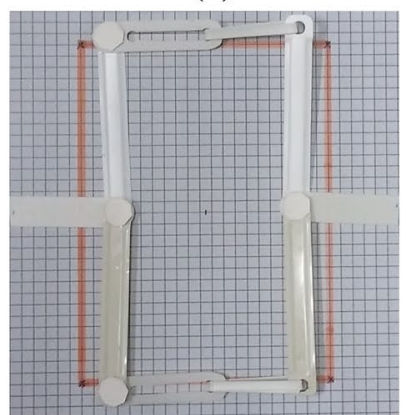

(g)
Fig. 8 Unit cell of the hybrid rhombic-re-entrant metamaterial a before deformation, $\mathbf{b} \varepsilon_{x}=-0.042, \mathbf{c} \varepsilon_{x}=0.239$, d $\varepsilon_{x}=-0.125$, e $\varepsilon_{x}=0.516, \mathbf{f} \varepsilon_{x}=-0.333$, and $\mathbf{g} \varepsilon_{x}=0.774$

deviation of the experimental results from the theoretical results. The deviation at larger strain may also be attributed to the infinitesimal strain assumption in the analysis. Notwithstanding this, reasonable agreement is observed for the latter at small strains.

\subsection{Hybrid rhombic-re-entrant metamaterial}

Perusal to Eqs. (9) and (13) for the Poisson's ratio $v_{x y}$ of the hybrid rhombic-re-entrant metamaterial, as a result from $\sigma_{x}$ loading, indicates that the magnitudes of $v_{x y}$ is gigantic if the inclined rod is highly aligned to the $x$-axis, i.e.

$\lim _{\theta \rightarrow 0^{\circ}} v_{x y}=\left\{\begin{array}{l}+\infty \Leftrightarrow \sigma_{x}<0 \\ -\infty \Leftrightarrow \sigma_{x}>0\end{array}\right.$

but diminishes when the inclined rod becomes oriented towards the $y$-axis

$\lim _{\theta \rightarrow 90^{\circ}} v_{x y}=0$.

The variation of Poisson's ratio for other values of $\theta$ are plotted in Fig. 11 to aid visual observation.

\subsection{Hybrid kite-arrowhead metamaterial}

With reference to Eqs. (26) and (42) for the Poisson's ratio $v_{x y}$ of the hybrid kite-arrowhead metamaterial arising from $\sigma_{x}$ loading, we observe that

$\lim _{\theta_{1} \rightarrow 0^{\circ}} v_{x y}=\left\{\begin{array}{l}+\infty \Leftrightarrow \sigma_{x}<0 \\ -\infty \Leftrightarrow \sigma_{x}>0\end{array}\right.$

and

$\lim _{\theta_{1} \rightarrow 90^{\circ}} v_{x y}=0 \Leftrightarrow \sigma_{x}<0$,

which resemble the hybrid rhombic-re-entrant metamaterial characteristic. Unlike the hybrid rhombic-reentrant metamaterial, perusal to Eq. (42) for the hybrid kite-arrowhead metamaterial under tensile load further indicates that

$\lim _{\theta_{1} \rightarrow \theta_{2}} v_{x y}=-\infty \Leftrightarrow \sigma_{x}>0$.

In addition to these extremes, the Poisson's ratio for other values of $\theta_{1}$ are furnished in Figs. 12 and 13 to facilitate visual observation under compressive and tensile $\sigma_{x}$, respectively.

For Special Case I where the joints ABC in Fig. 7a form the corners of an isosceles triangle such that $I_{3}=I_{2}\left(\operatorname{or} \theta_{3}=\theta_{2}\right)$ as the original condition, Eq. (42) reduces to

$v_{x y}=\frac{1}{\tan \theta_{1}-\tan \theta_{2}}\left(\frac{1}{\tan \theta_{1}}+\frac{1}{\tan \theta_{2}}\right)$.

To facilitate comparison with Eq. (48), one may express Eq. (26) as

$v_{x y}=\frac{1}{\tan \theta_{1}+\tan \theta_{3}}\left(\frac{1}{\tan \theta_{1}}+\frac{1}{\tan \theta_{3}}\right)$. 
Fig. 9 Unit cell of the hybrid kite-arrowhead metamaterial a before deformation, $\mathbf{b}$ $\varepsilon_{x}=-0.0196, \mathbf{c} \varepsilon_{x}=0.0327, \mathbf{d}$ $\varepsilon_{x}=-0.0588, \mathbf{e} \varepsilon_{x}=0.0588$, $\mathbf{f} \varepsilon_{x}=-0.0980$, and $\mathbf{g}$ $\varepsilon_{x}=0.0850$

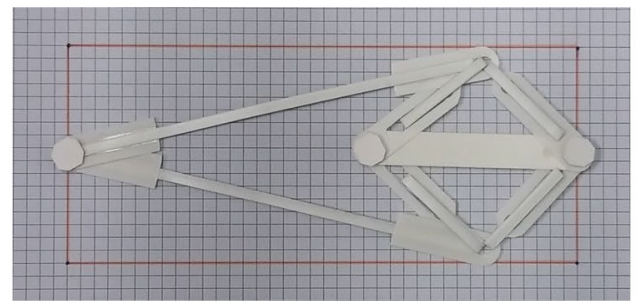

(a)

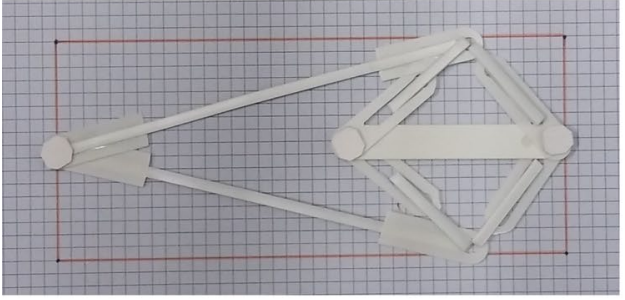

(b)

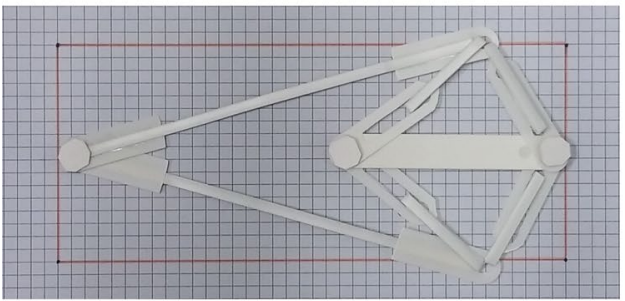

(d)

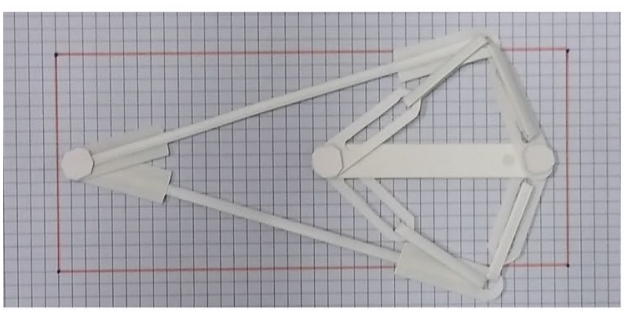

(f)

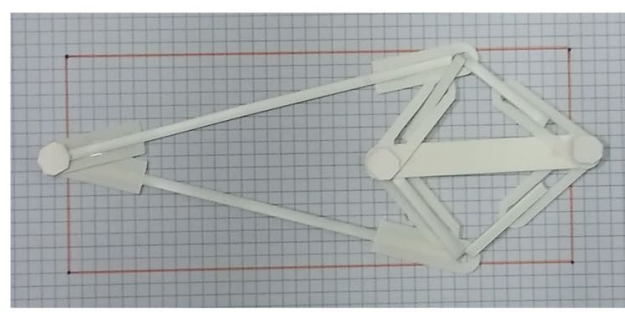

(c)

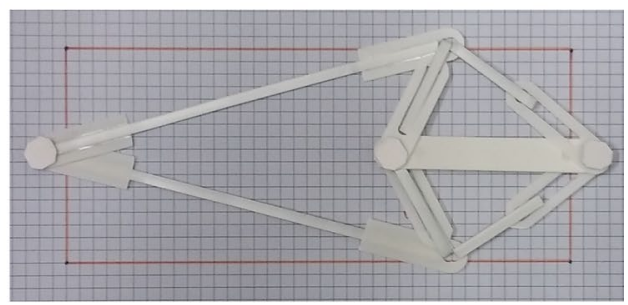

(e)

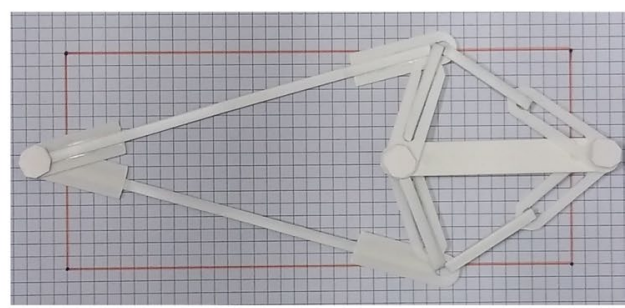

(g)
For Special Case II where the angle BAC in Fig. 7a forms a right angle, we have $\theta_{2}+\theta_{3}=90^{\circ}$ such that applying the relation $\tan \theta_{2} \tan \theta_{3}=1$ on Eq. (42) gives $v_{x y}=-\tan \left(\theta_{1}+\theta_{3}\right) / \tan \theta_{1}$ or

$v_{x y}=\frac{1}{\tan \theta_{1}} \frac{1}{\tan \left(\theta_{1}-\theta_{2}\right)}$,

which is comparable to Eq. (26). The Poisson's ratio plots of these two special cases are shown in Fig. 14.

\section{Applications}

For the purposes of wrapping a flat sheet onto a curved surface, a positive, a zero or a negative Poisson's ratio material is advised if the surface takes the form of an anticlastic shape, a cylindrical shape or a synclastic shape in order to reduce bending stress in the sheet material. This line of reasoning implies that the choice of Poisson's ratio sign is dependent on the application. Likewise, the choice 


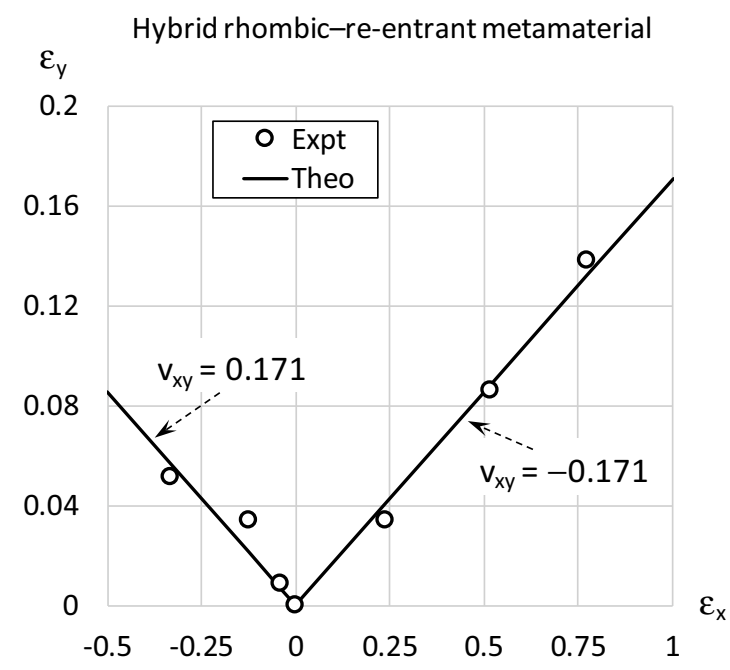

(a)

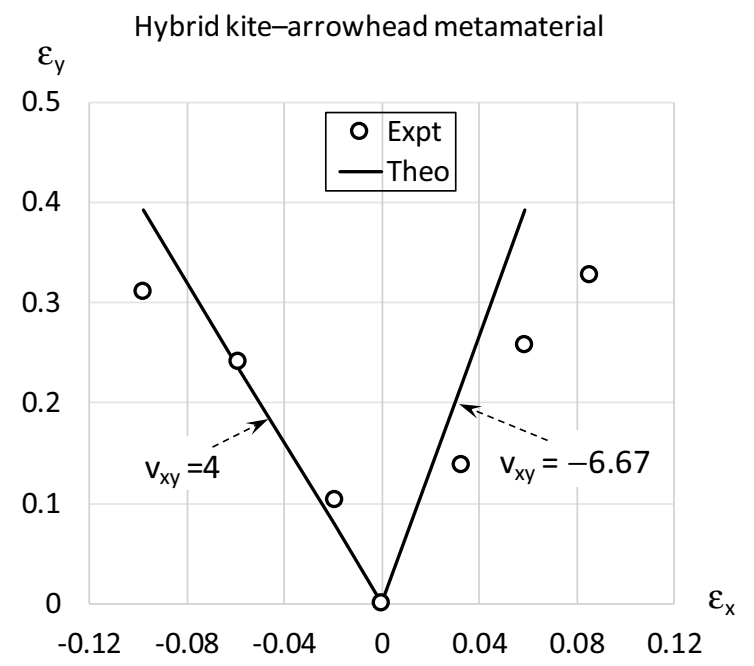

(b)

Fig. 10 Comparison between experimental and theoretical strains for the a hybrid rhombic-re-entrant, and the $\mathbf{b}$ hybrid kite-arrowhead metamaterials

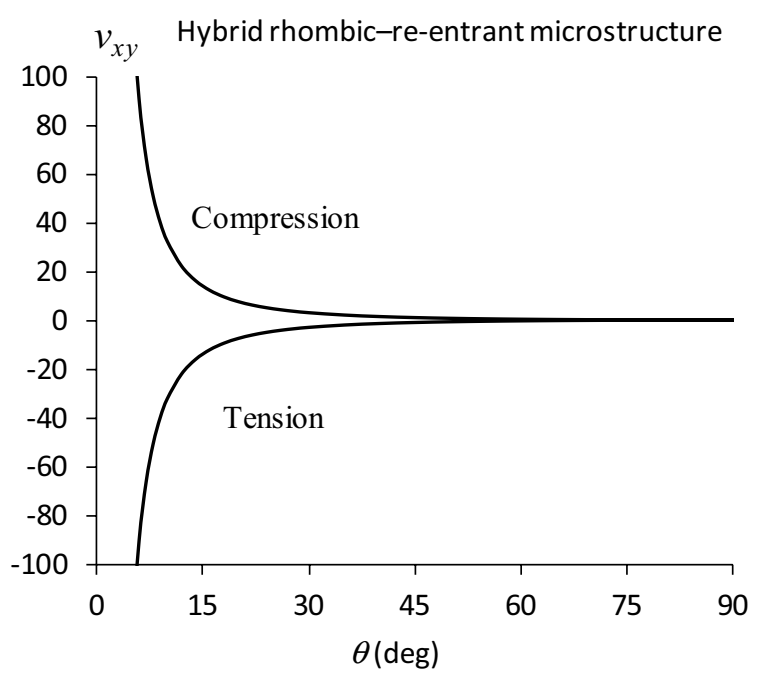

Fig. 11 Switching of Poisson's ratio $v_{x y}$ sign for the hybrid rhombicre-entrant metamaterial due to $\sigma_{x}$ direction reversal

of auxetic fiber is useful to resist fiber pull-out from the matrix material due to the self-locking mechanism in the form of radial expansion during axial pulling; however, auxetic fibers are easily pushed out from matrix material due to radial contraction. In fact, it is the conventional fibers that resist push-out due to radial expansion, although it is also known that conventional fibers are easily pulled out due to the resulting radial contraction. If the fiber behaves as auxetic material during fiber pull-out $\left(\sigma_{z}>0 \Rightarrow v_{z r}<0\right)$ but becomes conventional material during fiber push-out

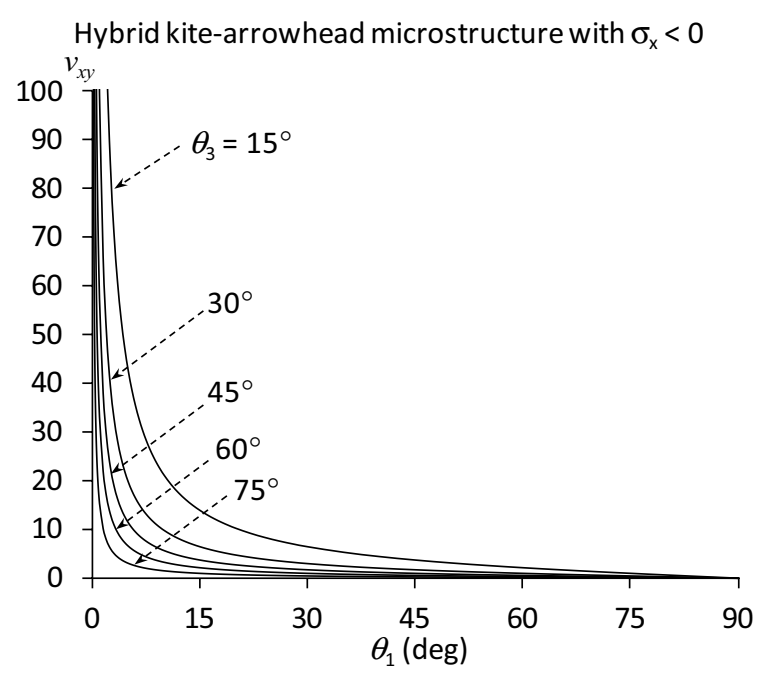

Fig. 12 Positive Poisson's ratio $v_{x y}$ for the hybrid kite-arrowhead metamaterial under compressive $\sigma_{x}$

$\left(\sigma_{z}<0 \Rightarrow v_{z r}>0\right)$, then such a fiber resists both pull-out and push-out as a result of its duality.

\section{Conclusions}

Two metamaterial microstructures - the kite-arrowhead metamaterial and the hybrid kite-arrowhead metamaterial-have been introduced herein to produce sign toggling of Poisson's ratio upon reversal of applied stress 


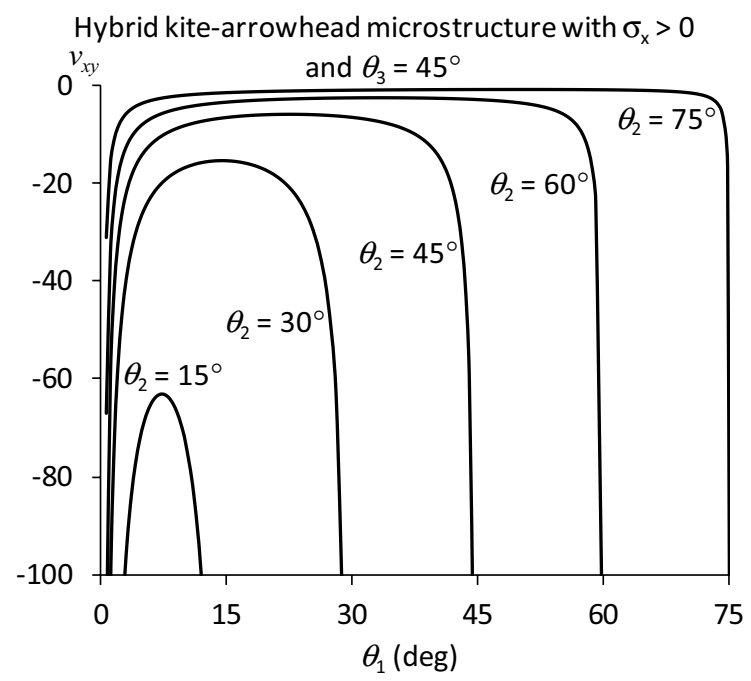

(a)

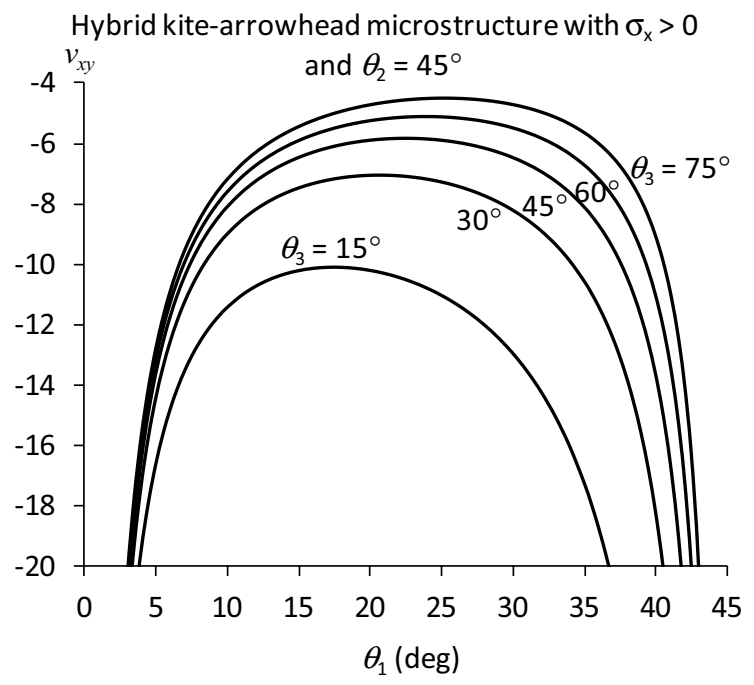

(b)

Fig. 13 Negative Poisson's ratio $v_{x y}$ for the hybrid kite-arrowhead metamaterial under tensile $\sigma_{x}$, with $\mathbf{a}$ variation of $\theta_{2}$ at fixed $\theta_{3}$, and $\mathbf{b}$ variation of $\theta_{3}$ at fixed $\theta_{2}$

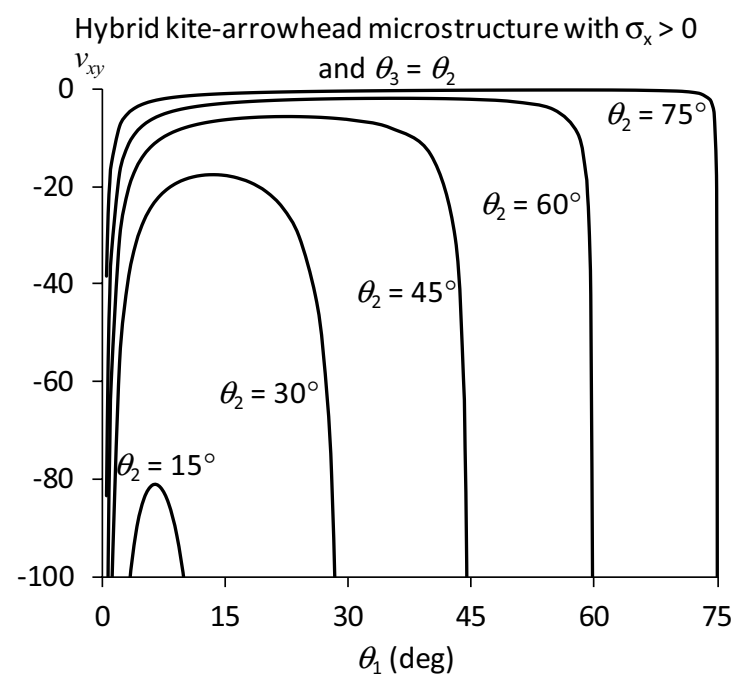

(a)

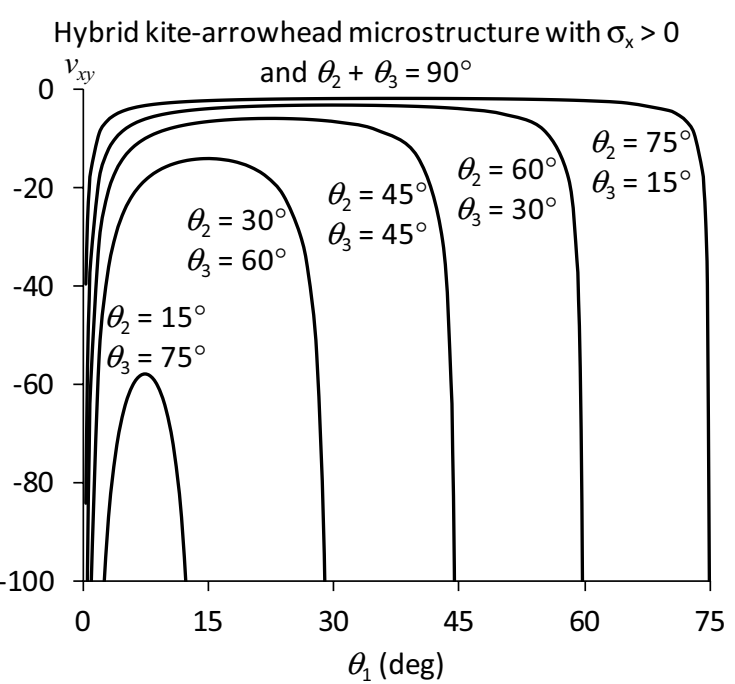

(b)

Fig. 14 Negative Poisson's ratio $v_{x y}$ for the hybrid kite-arrowhead metamaterial under tensile $\sigma_{x}$, under a Special Case I $\left(\theta_{3}=\theta_{2}\right)$, and $\mathbf{b}$ Special Case II $\left(\theta_{2}+\theta_{3}=90^{\circ}\right)$

direction. Specifically, these metamaterials exhibit the following opposing Poisson's ratio properties

- $\sigma_{x}<0 \Rightarrow v_{x y}>0$

- $\sigma_{x}>0 \Rightarrow v_{x y}<0$

or collectively as $v_{x y} \sigma_{x}<0$. While the previous approach of attaining Poisson's ratio sign switching upon stress reversal [48] can be achieved by catering for lateral contraction upon axial loading to give $v_{x y} \sigma_{x}>0$, the opposing sense of Poisson's ratio toggling elucidated herein is made possible by means of simultaneous jam and slide mechanism in both metamaterials. The capability of materials to switch their Poisson's ratio signs by mere stress direction reversal permits them to act in ways that can only be partially fulfilled by auxetic and conventional materials. The present metamaterials with Poisson's ratio sign toggling capability $v_{x y} \sigma_{x}<0$ not only complement the available conventional $v_{x y}>0$, auxetic $v_{x y}<0$, and recent Poisson's ratio sign switching $v_{x y} \sigma_{x}>0$ materials, but also avail wider options 
for the design of materials and structures to fulfil functions which until this time cannot be fully attained.

\section{Compliance with ethical standards}

Conflict of interest The corresponding author states that there is no conflict of interest.

\section{References}

1. Gibson LJ, Ashby MF (1982) The mechanics of three-dimensional cellular materials. Proc R Soc Lond A 382:43-59

2. Wojciechowski KW (1987) Constant thermodynamic tension Monte Carlo studies of elastic properties of a two-dimensional system of hard cyclic hexamers. Mol Phys 61:1247-1258

3. Lakes R (1987) Foam structures with a negative Poisson's ratio. Science 235:1038-1040

4. Caddock BD, Evans KE (1989) Microporous materials with negative Poisson's ratios. II. Mechanisms and interpretation. J Phys D Appl Phys 22:1877-1882

5. Alderson A, Evans KE (1995) Microstructural modelling of auxetic microporous polymers. J Mater Sci 30:3319-3332

6. Scarpa F, Tomlinson GR (1998) Vibroacoustics and damping analysis of negative Poisson's ratio honeycombs. Proc SPIE 3327:339-348

7. Smith CW, Grima JN, Caddock BD, Wootton RJ, Evans KE (1999) Microstructures producing negative Poisson's ratios: experiment and models. In: SEM annual conference on theoretical, experimental and computational mechanics, pp 184-187

8. Landau LD, Lifshitz EM (1959) Theory of elasticity. Pergamon Press, London

9. Fung YC (1965) Foundations of solid mechanics. Prentice-Hall, New Jersey

10. Ting TCT, Barnett DM (2005) Negative Poisson's ratios in anisotropic linear elastic media. J Appl Mech 72:929-931

11. Ting TCT, Chen T (2005) Poisson's ratio for anisotropic elastic materials can have no bounds. Q J Mech Appl Mech 58:73-82

12. Evans KE (1991) Auxetic polymers: a new range of materials. Endeavour 15:170-174

13. Bertoldi K, Reis PM, Willshaw S, Mullin T (2010) Negative Poisson's ratio behavior induced by an elastic instability. Adv Mater 22:361-366

14. Smith WA (1991) Optimizing electromechanical coupling in piezeocomposites using polymers with negative Poisson's ratio. Proc IEEE Ultrasonics Symp 1:661-666

15. Xu B, Arias F, Brittain ST, Zhao XM, Grzybowski B, Torquato S, Whitesides GM (1999) Making negative Poisson's ratio microstructures by soft lithography. Adv Mater 11:1186-1189

16. Alderson A, Rasburn J, Evans KE, Grima JN (2001) Auxetic polymeric filters display enhanced de-fouling and pressure compensation properties. Membr Technol 137:6-8

17. Lim TC, Acharya RU (2010) Performance evaluation of auxetic molecular sieves with re-entrant structures. J Biomed Nanotechnol 6:718-724

18. Evans KE, Alderson A (2000) Auxetic materials: functional materials and structures from lateral thinking! Adv Mater 12:617-628

19. Choi JB, Lakes RS (1991) Design of a fastener based on negative Poisson's ratio foam. Cell Polym 10:205-212

20. Jacobs S, Coconnier C, DiMaio D, Scarpa F, Toso M, Martinez J (2012) Deployable auxetic shape memory alloy cellular antenna demonstrator: design, manufacturing and modal testing. Smart Mater Struct 21:075013
21. Wang Z, Hu H (2014) Auxetic materials and their potential applications in textiles. Text Res J 84:1600-1611

22. Lakes R (1993) Advances in negative Poisson's ratio materials. Adv Mater 5:293-296

23. Alderson A (1999) A triumph of lateral thought. Chem Ind 10:384-391

24. Evans KE, Alderson A (2000) Auxetic materials: the positive side of being negative. Eng Sci Educ J 9:148-154

25. Yang W, Li ZM, Shi W, Xie BH, Yang MB (2004) Review on auxetic materials. J Mater Sci 39:3269-3279

26. Alderson A, Alderson KL (2007) Auxetic materials. J Aerosp Eng 221:565-575

27. Liu Y, Hu H (2010) A review on auxetic structures and polymeric materials. Sci Res Essays 5:1052-1063

28. Greaves GN, Greer AL, Lakes RS, Rouxel T (2011) Poisson's ratio and modern materials. Nat Mater 10:823-837

29. Prawoto $Y$ (2012) Seeing auxetic materials from the mechanics point of view: a structural review on the negative Poisson's ratio. Comput Mater Sci 58:140-153

30. Critchley R, Corni I, Wharton JA, Walsh FC, Wood RJK, Stokes KR (2013) A review of the manufacture, mechanical properties and potential applications of auxetic foams. Phys Status Solidi B 250:1963-1982

31. Darja R, Tatjana R, Alenka PC (2013) Auxetic textiles. Acta Chim Slov 60:715-723

32. Carneiro VH, Meireles J, Puga H (2013) Auxetic materials-a review. Mater Sci Pol 31:561-571

33. Bhullar SK (2015) Three decades of auxetic polymers: a review. e-Polym 15:205-215

34. Novak N, Vesenjak M, Ren Z (2016) Auxetic cellular materials-a review. Stroj Vestn J Mech Eng 62:485-493

35. Saxena KK, Das R, Calius EP (2016) Three decades of auxetics research -materials with negative Poisson's ratio: a review. Adv Eng Mater 18:1847-1870

36. Jiang JW, Kim SY, Park HS (2016) Auxetic nanomaterials: recent progress and future development. Appl Phys Rev 3:041101

37. Lim TC (2017) Analogies across auxetic models based on deformation mechanism. Phys Status Solidi RRL 11:1600440

38. Ma P, Chang Y, Boakye A, Jiang G (2017) Review on the knitted structures with auxetic effect. J Text Inst 108:947-961

39. Kolken HMA, Zadpoor AA (2017) Auxetic mechanical metamaterials. RSC Adv 7:5111-5129

40. Park HS, Kim SY (2017) A perspective on auxetic nanomaterials. Nano Converg 4:10

41. Lakes RS (2017) Negative-Poisson's-ratio materials: auxetic solids. Ann Rev Mater Res 47:63-81

42. Papadopoulou A, Laucks J, Tibbits S (2017) Auxetic materials in design and architecture. Nat Rev Mater 2:17078

43. Duncan O, Shepherd T, Moroney C, Foster L, Venkatraman PD, Winwood K, Allen T, Alderson A (2018) Review of auxetic materials for sports applications: expanding options in comfort and protection. Appl Sci 8:941

44. Ren X, Das R, Tran P, Ngo TD, Xie YM (2018) Auxetic metamaterials and structures: a review. Smart Mater Struct 27:023001

45. Lim TC (2015) Auxetic materials and structures. Springer, Singapore

46. Cauchi R, Attard D, Grima JN (2013) On the mechanical properties of centro-symmetric honeycombs with T-shaped joints. Phys Status Solidi B 250:2002-2011

47. Lim TC (2018) Poisson's ratio sign reversal with stress sign reversal. In: Auxetics 2018 Abstract Book 44-45 (Sheffield, United Kingdom)

48. Lim TC (2019) Composite microstructures with Poisson's ratio sign switching upon stress reversal. Compos Struct 209:34-44 